\title{
DESIGN AND SIMULATION ANALYSIS OF AN INTEGRATED $X Y Z$ MICRO-ACTUATOR FOR CONTROLLING DISPLACEMENT OF A SCANNING PROBE
}

\author{
VAN Hieu Dang \\ Hanoi University of Science and Technology, International Training Institute for Materials Science, Hanoi, Vietnam, and \\ FPT University, Hanoi, Vietnam \\ VAN TAM LE \\ Hanoi University of Science and Technology, International Training Institute for Materials Science, Hanoi, Vietnam
} KAZUHIRO HANE

Tohoku University, Department of Finemechanics, Sendai, Japan

Manh Hoang Chu

Hanoi University of Science and Technology, International Training Institute for Materials Science, Hanoi, Vietnam e-mail: hoangcm@itims.edu.vn; hoang.chumanh@hust.edu.vn

\begin{abstract}
We report an integrated micro-actuator for independent control of the displacement in three orthogonal directions. Electrostatic comb drives are employed for controlling the displacement in the $x$ and $y$ directions while a parallel plate-type actuation is used for driving the displacement in the $z$ direction. The three actuators are mechanically coupled, but are electrically isolated. The calculation models are established for investigating the operation characteristics of the micro-actuator. The calculated results are in good agreement with those obtained by the finite element method in Comsol Multiphysics 4.3. The results of modal analysis show that the displacement in the three orthogonal directions can be independently controlled with low mode cross-talk.
\end{abstract}

Keywords: integrated $x y z$ micro-actuator, mode cross-talk, mechanical coupling bridge, electrical isolation trench

\section{Introduction}

Recently, micro-actuators are of great interest for a variety of applications such as in nanopositioning, oscillating performance, mechanical sensors and scanning probe lithography (Liu et al., 2007; Legtenberg et al., 1996; Sasaki et al., 2008; Huo et al., 2010; Hu et al., 2017). Micro-actuators can be one-, two- or multi-dimensional (Liu et al., 2007; Legtenberg et al., 1996; Sasaki et al., 2008). To drive a structure in a certain direction, a micro-actuator has to be constructed from springs, which is compliant to that motion direction. To drive a micro-actuator in one dimension in plane or out-of-plane, folded beam springs are often used because they can supply stable operation with large displacement (Liu et al., 2007; Legtenberg et al., 1996). For an $x y$ two-dimensional micro-actuator, one often employs springs used in one-dimensional micro-actuator and arranges them in two $x$ and $y$ orthogonal directions (Sasaki et al., 2008). There are also designs of $x y z$ three-dimensional micro-actuators reported in literature (Liu et al., 2007; Correa et al., 2016; Ando, 2004; Dong and Ferreira, 2009; Kim et al., 2014; Takahashi et al., 2009). In (Correa et al., 2016; Ando, 2004; Dong, 2009; Kim et al., 2014), springs for the xyz actuation are connected softly together or directly connected to the center plate without stiff gimbal frames, so the cross-axis coupling of motion still exists. An algorithm for compensating 
the coupled motion error was proposed in (Kim et al., 2014). To drive the $x y z$ actuator independently, topological configuration using electrical isolation trenches, stiction bars as an electrical jump wire, and mechanical coupling deploying stiff gimbal frames were introduced in (Takahashi et al., 2009). In this work, bidirectional lateral comb drives are used for the $x$ - and $y$-motions using flexural springs, whereas a unidirectional vertical comb drive is used for the $z$-motion using the torsion spring. To drive the $x y z$ micro-actuator, one can use actuation mechanisms such as piezoelectric, thermal or electrostatic actuation (Liu et al., 2007; Legtenberg et al., 1996; Sasaki et al., 2008; Correa et al., 2016; Ando, 2004; Dong, 2009; Kim et al., 2014; Takahashi et al., 2009; $\mathrm{Xu}$ et al., 2006; Wang et al., 2004). In these actuation mechanisms, electrostatic actuation is preferred for operation with low power consumption due to capacitance-based actuation without current. On the other hand, much effort is made to improve mechanical structures for sensing applications with high precision (Trinh et al., 2013; Weinberg and Kourepenis, 2006; Acar and Shkel, 2009), however, there are no many reports on the $x y z$ micro-actuator that has a mechanically decoupled structure for easy and precise control of the displacements. Moreover, a $x y z$ micro-actuator having the capability of independent control of the displacements and an easy fabrication process is necessary to report for applications in high precise positioning, low-noise sensing, and scanning probe lithography.

Therefore, we report here a $x y z$ micro-actuator whose its displacements in three orthogonal directions can be independently controlled. The operation characteristics of the micro-actuator are analytically investigated by solid and fluidic mechanical theory and electrostatic theory. The calculated results are also compared to those obtained by the finite element method.

\section{Design of $x y z$ micro-actuator}

The concept of the proposed $x y z$ micro-actuator is shown in Fig. 1a. The micro-actuator is constructed of two components, an $x y$ actuator consisting of electrostatic comb drives, comb drives 1 and 2 for controlling the $x$-axis displacement, comb drives 3 and 4 for controlling the $y$-axis displacement, and a parallel-plate electrostatic actuator ( $z$-axis actuator) for controlling the out-of-plane ( $z$-axis) displacement (Figs. 1b and 1c). The center plate can be independetly drived in the $x$ and $y$ axes by the $x y$ actuator. This is obtained by designing springs, which is compliant to one motion direction. These springs are then orthogonally designed which respond to displacements along the $x$ and $y$ axes. In this work, we employ folded beam springs, see Fig. 1d, which supplies stable operation with large displacements (Legtenberg et al., 1996). The $z$-axis actuator consists of a center plate that is suspended by extended crab-leg beam springs, Fig. 1c. The comb drives are mechanically coupled, but isolated electrically by separated gaps using insulation bridges, Fig. 1e, (Chu et al., 2011). The comb drives are designed on the fixed and movable suspending frames. In this work, we propose to directly integrate the counter electrode at the backside of the center plate to form a parrallel-plate capacitive actuator which can be fabricated by the silicon-on-insulator technology on a single chip. This will reduce effort in fabricating $x y z$ micro-actuators using a complicated assembly process as reported in (Liu et al., 2007). To supply a potential to the counter electrode, we use the contact hole as shown in Fig. 1f.

To drive the $x y z$ micro-actuator under DC voltage, we can establish a common ground line for the comb drives and the plate electrode using a folded beam spring of the $x$-axis actuator. The remaining electrodes use three remaining folded beam springs of the $x$-axis actuator for supplying voltages. However, to dynamically drive the $x y z$ actuator, more electric interconnection lines are required. To effectively use the actuation of both sides of the $x y$ actuator, three electrodes are required for each axis, $x$ or $y$ (Chu et al., 2011). Therefore, we propose an $x y z$ micro-actuator with folded beam springs modified in Fig. 2, in which the four folded springs in Fig. 1 devided 


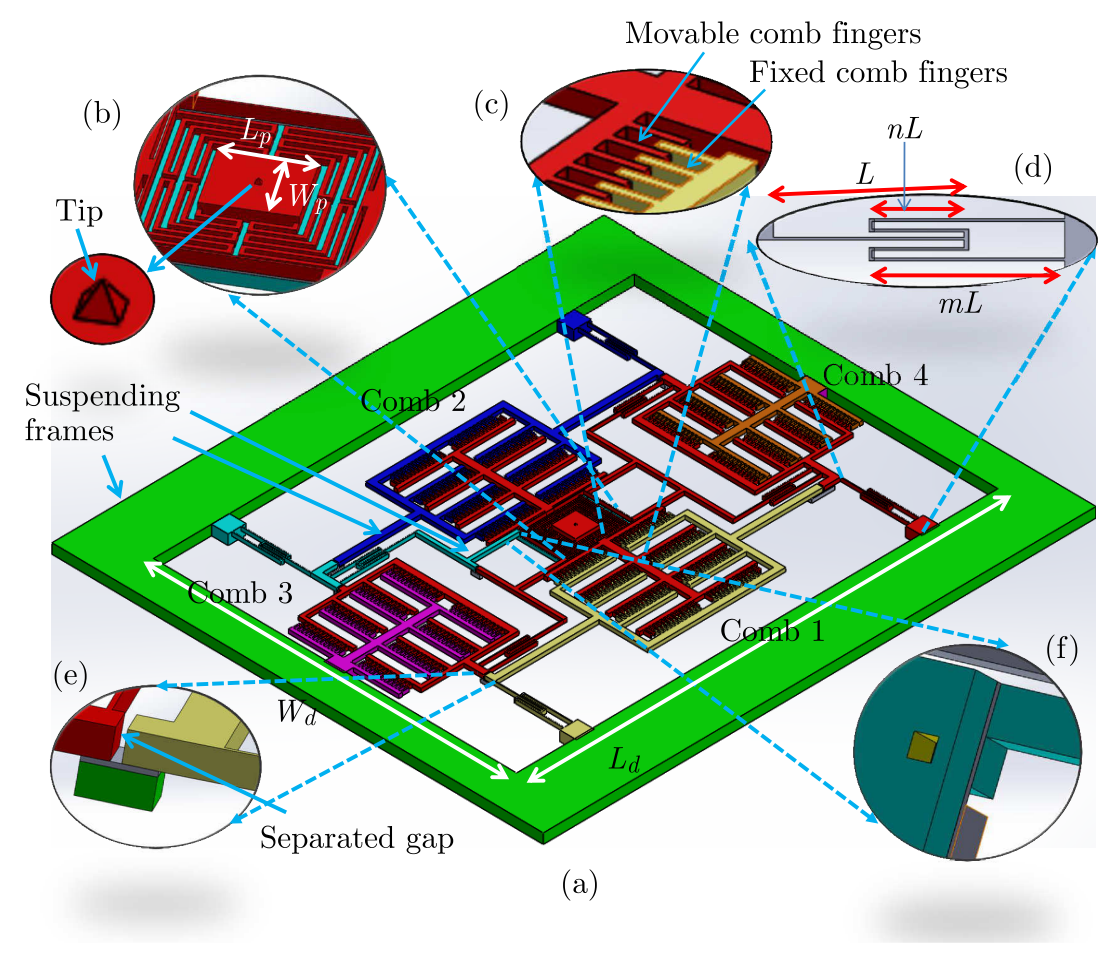

Fig. 1. Three-dimensional schematic view of the $x y z$ micro-actuator (a) and magnified images of interdigitated comb drives (b), z-axis actuator (c), folded springs (d), mechanically coupled but electrically isolated bridge (e), and contact hole for electrically interconnecting between conductive lines on the upper layer and under electrode (back-side counter electrode) (f)

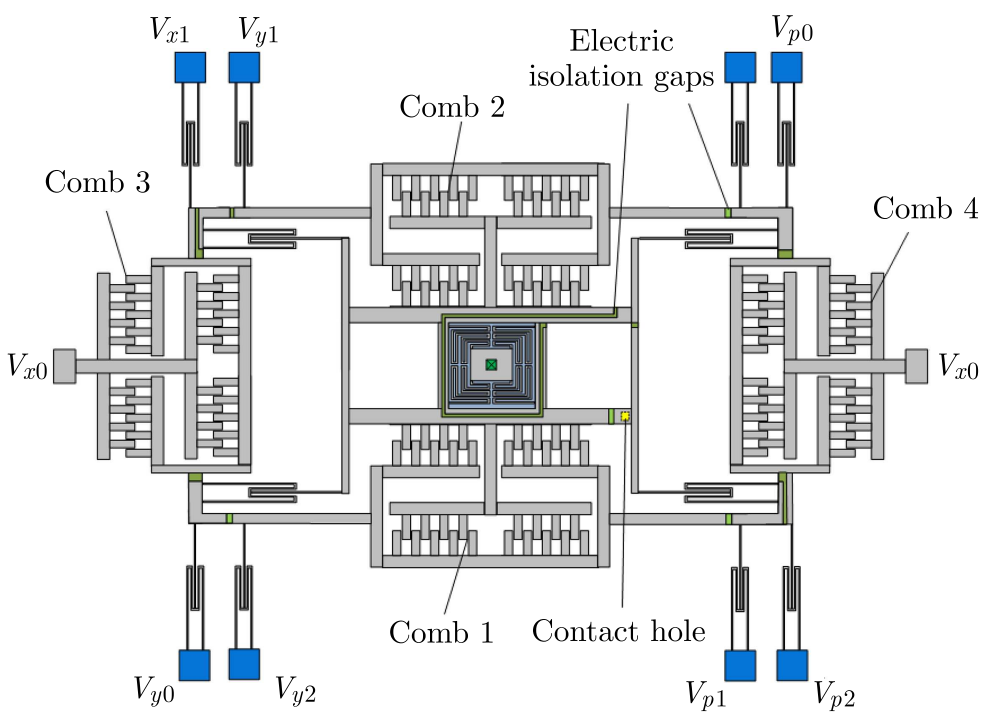

Fig. 2. Diagram of electric interconnection for driving the $x y z$ micro-actuator independently

into eight folded beam springs. Electrodes, $V_{x 0}\left(V_{y 0}\right), V_{x 1}\left(V_{y 1}\right)$ and $V_{x 2}\left(V_{y 2}\right)$ are for driving the center plate along the $x(y)$ axis while two electrodes, $V_{p 0}$ and $V_{p 1}$ are for supplying voltage for the $z$-axis actuation. Using the three driving electrodes for the $x$ and $y$ actuator, we can use a differential driving mechanism to enhance the $x$ and $y$ displacements and the linear dependence between the controlling voltage and the displacements (Chu et al., 2011). 


\section{Analysis model}

To investigate the operation characteristics of the $x y z$ micro-actuator, calculation and numerical methods are used, and several assumptions for simplifying the calculations are given. The micro-actuator is asumed to be made of single crystal silicon. The assumptions of the kinematic theory conform to the Bernoulli-Euler beam theory. The properties of the air operation medium and single crystal silicon are shown in Table 1. In the following, we will present models for investigating the operation characteristics of the micro-actuator.

Table 1. Physical properties of the air operation medium and single crystal silicon

\begin{tabular}{|l|c|c|}
\hline \multicolumn{1}{|c|}{ Properties } & Air & Silicon \\
\hline \hline Density $\left[\mathrm{kg} / \mathrm{m}^{3}\right]$ & 1.2 & 2329 \\
\hline Elastic modulus $\left[\mathrm{N} / \mathrm{m}^{2}\right]$ & & $170 \cdot 10^{9}$ \\
\hline Poisson ratio & & 0.28 \\
\hline Dielectric constant & 1 & 11.68 \\
\hline Viscous $\left[\mathrm{Ns} / \mathrm{m}^{2}\right]$ & $1.8 \cdot 10^{-5}$ & \\
\hline
\end{tabular}

\subsection{The $z$-axis actuator}

As presented in Fig. 1, the $x y z$ micro-actuator uses a parallel-plate capacitive actuator for controlling the $z$-axis displacement of the probe. The center plate of the $z$-axis actuator is connected to the $x y$ actuator by four extended crab-leg beam springs. The counter electrode is designed at the backside of the center plate for forming a parallel-plate capacitive actuation. To investigate the operation of this actuator, a single degree of freedom mass-spring system model is used. The movable part consists of the center plate, four springs having the effective mass $m_{e q}$ and a damper. The four spring system has the effective stiffness $k_{z}$. The damper is to describe damped vibration caused by the squeeze film air damping effect (Chu, 2016; Bao, 2005). The resonant angular frequency of this actuator $\omega_{z}$ is found from

$$
\omega_{z}=\sqrt{\frac{k_{z}}{M_{z}}}
$$

in which $M_{z}$ is the sum of the effective mass $m_{e q}$ and mass of the center plate. $m_{e q}\left(=13 m_{b} / 35\right)$ is the equivalent mass of the beam derived by the Rayleigh method (Gere and Timoshenko, 1997), in which $m_{b}$ is mass of the beam.

The extended crab-leg beam spring is shown in Fig. 3a. For convenience, in deriving the expression of the stiffness, we interporate the spring unit as in Fig. 3b. We can divide the spring into three parts, fixed-guided beams with lengths $l_{a}$ and $l_{b}$, and three crab-leg spring units with lengths $\left(l_{c 11}, l_{c 12}\right),\left(l_{c 21}, l_{c 22}\right)$, and $\left(l_{c 31}, l_{c 32}\right)$. The stiffness of the fixed-guided beams $l_{a, b}$ is

$$
K_{a, b}=\frac{E w_{c s} t_{z}^{3}}{4 l_{a, b}^{3}}
$$

where $w_{c s}$ and $t_{z}$ are width and thickness of the extended crab-leg beam spring.

The stiffness of the crab-leg spring unit is calculated by (Beer et al., 2003; Liu, 2011)

$$
K_{c}=\frac{1}{\frac{\left(l_{1}-K_{2}\right)^{2} l_{2}}{G I_{p}}+\frac{l_{1}^{3}+l_{2}^{3}+3 K_{2} l_{1}\left(K_{2}-l_{1}\right)}{3 E I_{1}}}
$$

where $l_{1}$ and $l_{2}$ are lengths of the crab-leg beam unit, Fig. 3b, and

$$
K_{2}=-\frac{K_{2 P}}{\delta_{2 P}} \quad K_{2 P}=\frac{l_{1}^{2}}{2 E I_{1}}-\frac{l_{1} l_{2}}{G I_{P}} \quad \delta_{2 P}=\frac{l_{1}}{E I_{1}}-\frac{l_{2}}{G I_{P}}
$$




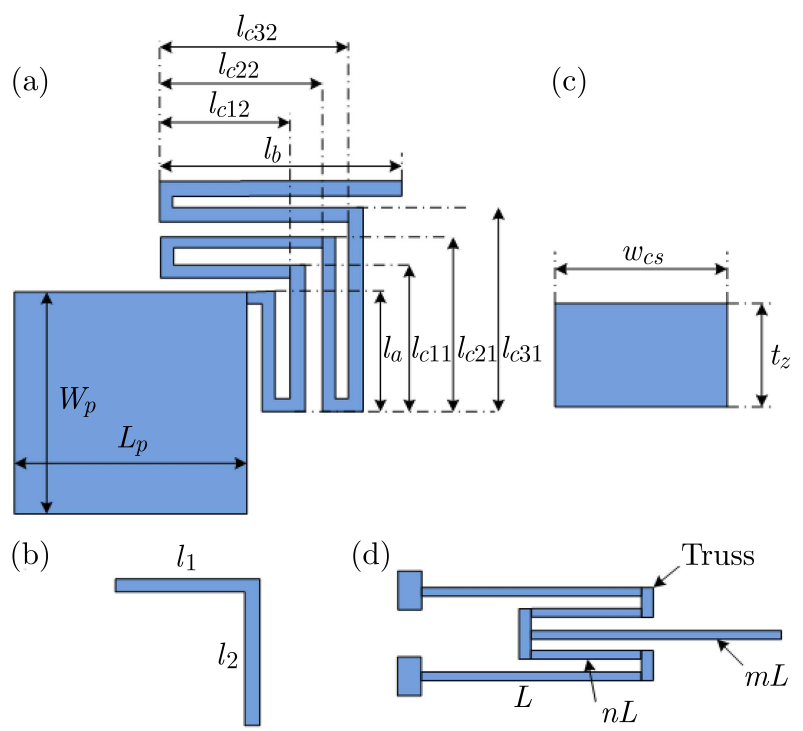

Fig. 3. Schematic drawing of springs: (a) extended crab-leg beams spring for the $z$-axis actuator,

(b) simplified crab-leg spring unit with defined dimensions and (c) folded beam spring for the $x y$ actuator

Here $I_{P}$ is the polar moment of inertia of the cross-sectional area of the crab-leg beam given by

$$
I_{P}=\frac{1}{16}\left[w_{c s} t_{z}^{3}\left(\frac{16}{3}-3.36 \frac{t_{z}}{w_{c s}}\right)\right]
$$

where $G(=E /[2(1+\nu)])$ is shear modulus in which $\nu$ is Poisson's ratio of silicon, and $I_{1}\left(=w_{c s} t_{z}^{3} / 12\right)$ is the inertial moment of the cross-section about its neutral axis.

Thus, the total stiffness of the extended crab-leg beam spring can be calculated by

$$
k_{z}=\frac{1}{\frac{1}{K_{a}}+\frac{1}{K_{c 1}}+\frac{1}{K_{c 2}}+\frac{1}{K_{c 3}}+\frac{1}{K_{b}}}
$$

Here, $K_{c 1}, K_{c 2}$, and $K_{c 3}$ are stiffnesses of the three crab-leg beam units, Fig. 3a, which are found from Eq. (3.3).

The electrostatic force acting on the center plate (the movable electrode) is evaluated by

$$
F_{\text {ele }}=-\frac{1}{2} \varepsilon_{o} \frac{V^{2} A}{\left(g_{z}-d_{z}\right)^{2}}
$$

where $A$ is the electrode overlap area, $V$ is the applied voltage, $g_{z}$ is the gap between two electrodes and $d_{z}$ is the vertical displacement. The displacement of the movable electrode can be controlled by applying voltage to the actuation electrodes. The minus means that the electrostatic force is always an attractive force. Under a DC driving condition, the displacement $d_{z}$ along the $z$-axis can be derived based on the balance between the electrostatic force and the elastic force as follows

$$
k_{z} d_{z}=\frac{1}{2} \varepsilon_{o} \frac{V_{d c}^{2} A}{\left(g_{z}-d_{z}\right)^{2}}
$$

To solve this equation, we can use a numerical method.

In the parallel-plate actuation, there is a static equilibrium position $\left(V_{a c}=0\right)$ where the electrostatic force balances with the elastic force. The DC applied voltage at this critical state is called to be $V_{\text {pull-in }}$ at $d_{z}=g_{z} / 3$ (Bao and Yang, 2007)

$$
V_{\text {pull-in }}=\sqrt{\frac{8}{27} \frac{k_{z} g_{z}^{3}}{\varepsilon_{o} A}}
$$


when $V_{d c}$ is larger than or equal to $V_{p u l l-i n}$, the equilibrium position does not exist, the probe will be at an unstable state and can contact or snap with the counter electrode. So, for the electrostatic actuator used in the scanning probe, the operating voltage of the actuator must be smaller than $V_{\text {pull-in }}$.

\subsection{The $x y$ actuators}

The displacement along the $x$ and $y$ axes is drived by interdigitated comb drives (Fig. 1). The movable combs are suspended on the folded beam springs with stiffness $k_{x, y}$. The displacement of the center plate along the $x$ and $y$ axes actuated by the lateral force $F_{l}\left(=n_{c} \varepsilon_{o} t V^{2} / g_{c}\right)$ is calculated by (Legtenberg et al., 1996)

$$
x, y=\frac{2 n_{c} \varepsilon_{o} t_{x y}}{k_{x, y} g_{c}} V_{d c}^{2}
$$

where $n_{c}$ is the number of the comb fingers, $t_{x y}$ is thickness of the comb fingers, $x(y)$ is the displacement of the center plate along the $x(y)$ axis, $g_{c}$ is the gap between two adjacent fingers. In investigations, $t_{x y}$ is equal to thickness $t$ of the $x y z$ micro-actuator, while $t_{z}$ can be different from $t_{x y}$. This choice is dependent on design requirements of the device.

As presented, the folded beam spring is used for suspending the frames moving in the $x$ and $y$ axes. In this study, the folded beam springs in the two axes are identical. The stiffness of the folded beam spring in the $x$ and $y$ direction is derived based on the stiffness of folded-beam spring components connected in series and parallel as

$$
k_{x, y}=\frac{2 E w_{f b}^{3} t_{x y}}{\left(1+2 m^{3}+n^{3}\right) L^{3}}
$$

where $m$ and $n$ are multiples of $L$ showing length of the folded-beam spring components as shown in Fig. 3d. In this study, $m=1$ and $n=0.5$. The dimensional parameters of the plate, springs and comb fingers are shown in Table 2 .

Table 2. Dimensional parameters of the $x y z$ micro-actuator

\begin{tabular}{|l|c|c|c|}
\hline \multicolumn{1}{|c|}{ Structures } & $\begin{array}{c}\text { Length } \\
{[\mu \mathrm{m}]}\end{array}$ & $\begin{array}{c}\text { Width } \\
{[\mu \mathrm{m}]}\end{array}$ & $\begin{array}{c}\text { Height } \\
{[\mu \mathrm{m}]}\end{array}$ \\
\hline \hline Plate $\left(L_{p} \times W_{p} \times t\right)$ & 200 & 200 & 30 \\
\hline Tip $\left({ }_{L} t \times W_{t} \times h_{t}\right)$ & 14 & 14 & 10 \\
\hline Comb finger $\left(L_{c} \times W_{c} \times t\right)$ & 100 & 5 & $10-30$ \\
\hline Beam $L\left(L_{L} \times W_{L} \times t\right)$ & $100-600$ & $5-15$ & $10-30$ \\
\hline Total size of device $\left(L_{d} \times W_{d} \times t\right)$ & 4210 & 5060 & $10-30$ \\
\hline
\end{tabular}

\subsection{Air damping in the $x y z$ micro-actuator}

In the $x y z$ micro-actuator, there are two types of air damping, which depend on relative motion between structure components. When the structure components move perpendicularly to each other, squeeze film air damping is taken into consideration. This case is corresponding to the $z$-axis actuator. When the gap between two actuation electrodes of the $z$-axis actuator is small, the squeeze film air damping is a dominant energy dissipation mechanism in the $x y z$ micro-actuator. The damping ratio of the $z$-axis actuator $\zeta_{z}$ is given by (Bao, 2005)

$$
\zeta_{z}=\frac{\mu w_{p}^{4}}{0.84 m_{e q} g^{3} \omega_{z}}
$$


where $\omega_{z}$ is the angular frequency of the $z$-axis actuator, $w_{p}$ is width of the movable electrode (the center plate), $m_{e q}$ is the equivalent mass of the actuator, $\mu_{0}\left(=18.5 \cdot 10^{-5} \mathrm{Pas}\right)$ is the viscosity coefficient of the air. For low pressure, the effective viscosity coefficient is used (Veijola et al., 1998)

$$
\mu_{\text {eff }}=\frac{\mu_{0}}{1+9.658 K_{n}^{1.159}}
$$

where $K_{n}$ is the Knudsen number calculated by (Chu and Hane, 2011)

$$
K_{n}=\frac{\lambda}{d_{a}}
$$

Here $\lambda$ is the mean-free path and $d_{a}$ is the typical dimension of the device. The mean-free path is given by $\lambda=k T /\left(\sqrt{2} \pi P d^{2}\right)$ where $k\left(=1.38 \cdot 10^{-23} \mathrm{~J} / \mathrm{K}\right)$ is the Boltzman constant, $T$ is air temperature, $P$ is air pressure, and $d\left(=4.19 \cdot 10^{-10} \mathrm{~m}\right)$ is the collision diameter of gas molecules.

The remaining case is when the structure components are parallelly moving to each other, which corresponds to slide motion of the comb drives in the $x y$ actuators. In this case, slide film air damping is considered. The damping ratio $\zeta_{x, y}$ is calculated for motion in the $x$ and $y$ directions as follows

$$
\zeta_{(x, y)}=\frac{\mu A_{s}}{2 d \sqrt{m_{e f f}^{x, y} k_{x, y}}}
$$

where $A_{s}$ is the area of sidewalls of the overlap, and $m_{\text {eff }}^{x, y}$ are the effective masses of the actuator along the $x$ and $y$ vibration directions, respectively.

The quality factor of the $x y z$ micro-actuator in the $x, y$ and $z$ directions, $Q_{x, y, z}$ is calculated by (Bao, 2005)

$$
Q_{x, y, z}=\frac{1}{2 \xi_{x, y, z}}
$$

Thus, we have built a model for analyzing the operation characteristics of the $x y z$ micro-actuator. In the following, we will present results obtained by employing the above formulated equations. The calculation results are compared to those obtained from numerical simulation using Comsol Multiphysics 4.3. In this study, the used Comsol module is a MEMS module.

\section{Results and discussion}

The first three mode shapes and natural frequencies of the extended crab-leg beam spring-based $z$-axis actuator obtained from simulation are shown in Fig. 4. The parameters of the spring and the center plate are shown in Fig. 4 and Table 2. Here, we fixed the length of the crab-leg beam spring in investigations while changing its width and thickness. The first mode is the operation mode of the $z$-axis actuator. The natural frequencies of the first and second mode are $25.4 \mathrm{kHz}$ and $44.1 \mathrm{kHz}$, respectively. The natural frequency discrepancy between the first and second mode is $73.6 \%$, which satisfies the requirement of the low mode cross-talk (Trinh et al., 2013). The stiffness of the spring $k_{z}$ calculated by Eq. (3.6) is $88.9 \mathrm{~N} / \mathrm{m}$. The effective mass of the center plate and the extended crab-leg beams spring $M_{z}$ is $3.66 \cdot 10^{-9} \mathrm{~kg}$. The resonant frequency is calculated by Eq. (3.1) to be $24.8 \mathrm{kHz}$. The error between the calculation and simulation results is $2.4 \%$. So, the theoretical expressions can be used to optimize the operating frequency of the $z$-axis actuator.

For integrating the $z$-axis actuator in the $x y z$ actuator, we further investigate the mode cross-talk, pull-in voltage, and the resonant frequency depending on the width of the spring 
(a)

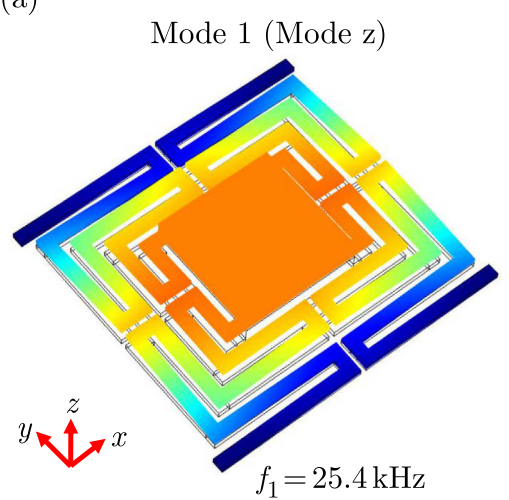

(b)

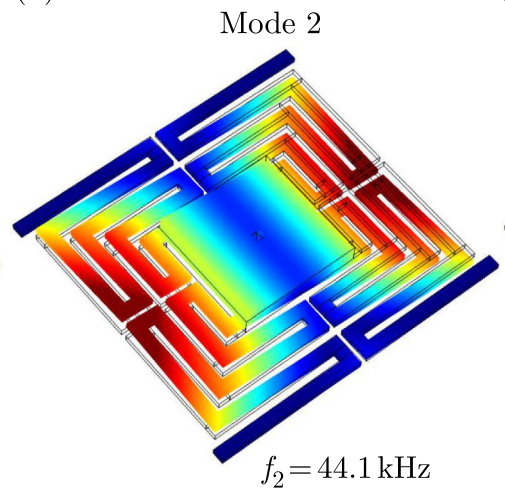

(c)

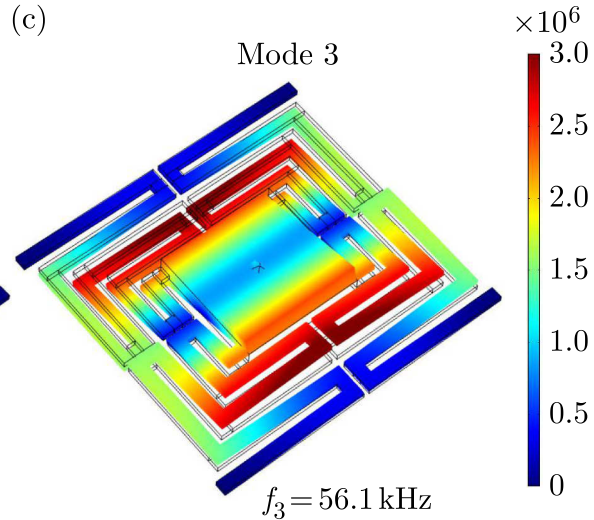

Fig. 4. The first three mode shapes and natural frequencies of the $z$-axis actuator are shown respectively in (a), (b), and (c). The dimensional parameters of the extended crab-leg beams spring are $l_{a}=230 \mu \mathrm{m}, l_{b}=95 \mu \mathrm{m}, l_{c 11}=160 \mu \mathrm{m}, l_{c 12}=215 \mu \mathrm{m}, l_{c 21}=145 \mu \mathrm{m}, l_{c 22}=160 \mu \mathrm{m}, l_{c 31}=95 \mu \mathrm{m}$, $l_{c 32}=145 \mu \mathrm{m}, w_{c s}=20 \mu \mathrm{m}$ and $t_{z}=10 \mu \mathrm{m}$

(a)

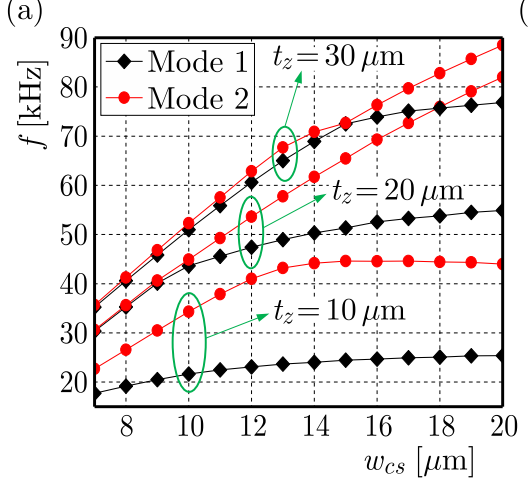

(b)

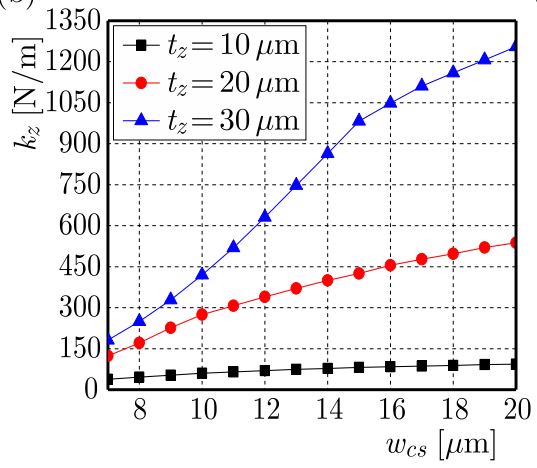

(c)

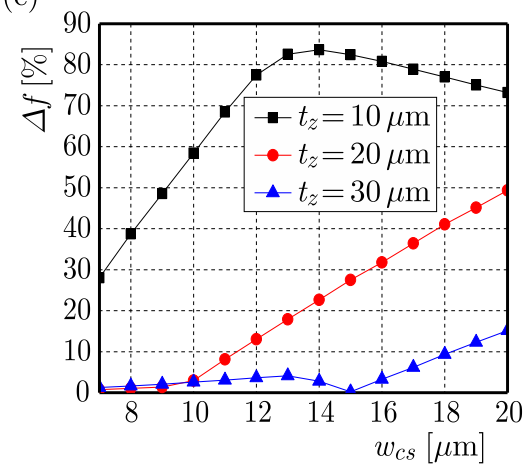

Fig. 5. (a) Frequency of modes 1 and 2, (b) stiffness of mode 1, and (c) frequency discrepancy between modes 1 and 2 of the $z$ actuator investigated as a function of width of extended crab-leg beam spring for three thicknesses of the spring beam, $t_{z}=10,20$ and $30 \mu \mathrm{m}$

beam for different device layer thicknesses. The frequency of modes 1 and 2 , stiffness of mode 1 , and frequency discrepancy between modes 1 and 2 of the $z$-axis actuator investigated as a function of width of the extended crab-leg beam spring for three thicknesses of the spring beam, $t_{z}=10 \mu \mathrm{m}, 20 \mu \mathrm{m}$ and $30 \mu \mathrm{m}$, are shown in Figs. 5a-5c, respectively. To obtain the stiffness $k_{z}$ of the $z$-axis actuator (mode 1) as shown in Fig. 5b, a semi-analytical method is used. $k_{z}$ is determined from the frequency $f_{z}$ of mode 1 and the effective mass $M_{z}$ by $k_{z}=\left(2 \pi f_{z}\right)^{2} M_{z}$. Thus, the operation frequency of the $z$-axis actuator increases with width of the spring beam. For thinner spring beams, $f_{z}$ is lower and has a narrower variation range of the operation frequency compared to that of thicker spring beams, Fig. 5a. For example, at $t_{z}=10 \mu \mathrm{m}, f_{z}$ is only modified by $40 \%$, from $17.7 \mathrm{kHz}$ to $25.4 \mathrm{kHz}$, while at $t_{z}=30 \mu \mathrm{m}, f_{z}$ is varied up to $118 \%$, from $35.3 \mathrm{kHz}$ to $76.9 \mathrm{kHz}$. The variation range of the operation frequency is chosen based on different applications. However, for thicker spring beams the frequency discrepancy $\delta f$ becomes smaller (Fig. 5c), which leads to the increment of the mode coupling in the $z$-axis actuator, meaning that the mode cross-talk is high. This can be explained that when thickness of the spring beam increases, the flexural modes in the plane become prior to the flexural mode in the $z$-direction. In general, to satisfy the requirement of the low mode cross-talk, the aspect ratio, $w_{c s} / t_{z}$, must be large enough. For example, at $t_{z}=10 \mu \mathrm{m}$, to obtain $\delta f$ larger than $40 \%$, $w_{c s} / t_{z}$ must be larger than 0.8 , while at $t_{z}=20 \mu \mathrm{m}, w_{c s} / t_{z}$ must be larger than 0.87 . In this study, we have kept the length of total spring beams to maintain the footprint of the device 
(Table 2) while its admired operation characteristics can be well modified by varying width and thickness of the spring beams.

The pull-in voltage $V_{\text {pull-in }}$ is investigated as a function of the beam width for three thickness values, $t_{z}=10 \mu \mathrm{m}, 20 \mu \mathrm{m}$ and $30 \mu \mathrm{m}$, as shown in Fig. 6 . The parameters range chosen for investigation is based on the criterion of practice fabrication that the width/thickness to length aspect ratio of the beam is less than 20 . Thus, when the beam width increases, $V_{\text {pull-in }}$ increases monotonically with $w_{c s}$. From these investigations, we can choose the applied voltage suitable for the $z$-axis actuator to avoid the sticking effect in the operation.

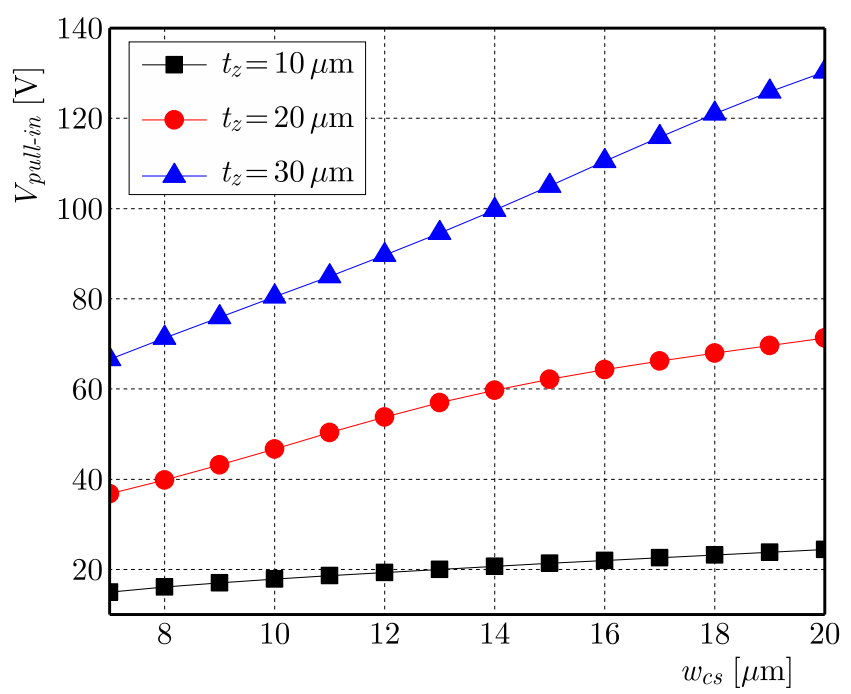

Fig. 6. Pull-in voltage of the $z$-axis actuator as a function of width of the extended crab-leg beam spring with the actuation gap, $g_{z}=3 \mu \mathrm{m}$, for three different thicknesses $t_{z}=10 \mu \mathrm{m}, 20 \mu \mathrm{m}$ and $30 \mu \mathrm{m}$

The mode shapes and natural frequencies of three operation modes of the $x y z$ micro-actuator are shown in Fig. 7. The two first modes are in-plane vibration modes in the $x$ and $y$ directions having the frequencies $f_{x}$ and $f_{y}$, respectively. The remaining mode is the out-of-plane vibration mode (the $z$-axis mode) having the frequency $f_{z}$. The resonant frequency values in the three vibration directions theoretically calculated using Eqs. (3.1), (3.6) and (3.11) are also shown in Table 3 . The errors between the calculated and simulated results for $f_{x}, f_{y}$, and $f_{z}$ are $2.9 \%, 7.2 \%$ and $2.3 \%$, respectively. To independently drive the micro-actuator along the $x, y$ and $z$ axes, the mode split has to be large enough to suppress the mode coupling (Trinh et al., 2013; Weinberg and Kourepenis, 2006; Fang et al., 2018, Acar and Shkel, 2009). The mode split between the first and second modes is $88.23 \%$, while these two modes are completely splitted with the third mode $(0.85 \mathrm{kHz}$ and $1.6 \mathrm{kHz}$ compared to $27.9 \mathrm{kHz})$. The operation frequencies and mode splits in the proposed $x y z$ micro-actuator are easily controlled by varying stiffness of the springs.

Table 3. Analytical and simulation results of natural frequencies of the $x y z$ micro-actuator

\begin{tabular}{|l|c|c|c|}
\hline & $f_{x}[\mathrm{kHz}]$ & $f_{y}[\mathrm{kHz}]$ & $f_{z}[\mathrm{kHz}]$ \\
\hline \hline Theoretical analysis & 0.87 & 1.51 & 24.82 \\
\hline FEM & 0.85 & 1.62 & 25.41 \\
\hline$\Delta[\%]$ & 2.9 & -7.2 & 2.3 \\
\hline
\end{tabular}

We have also investigated the resonant operation frequency of the three interested modes depending on the thickness to width aspect ratios of the spring beams, $t / w_{c s, f b}$, as shown in Fig. 8. The frequencies of the $x$ and $y$ in-plane modes are almost independent of the thickness $t_{x y}$. However, the frequency of the out-of-plane mode is dependent on the aspect ratio of $t_{z} / w_{c s}$. The frequency is linearly proportional to $t_{z} / w_{c s}$. To meet the operation requirement with the low 

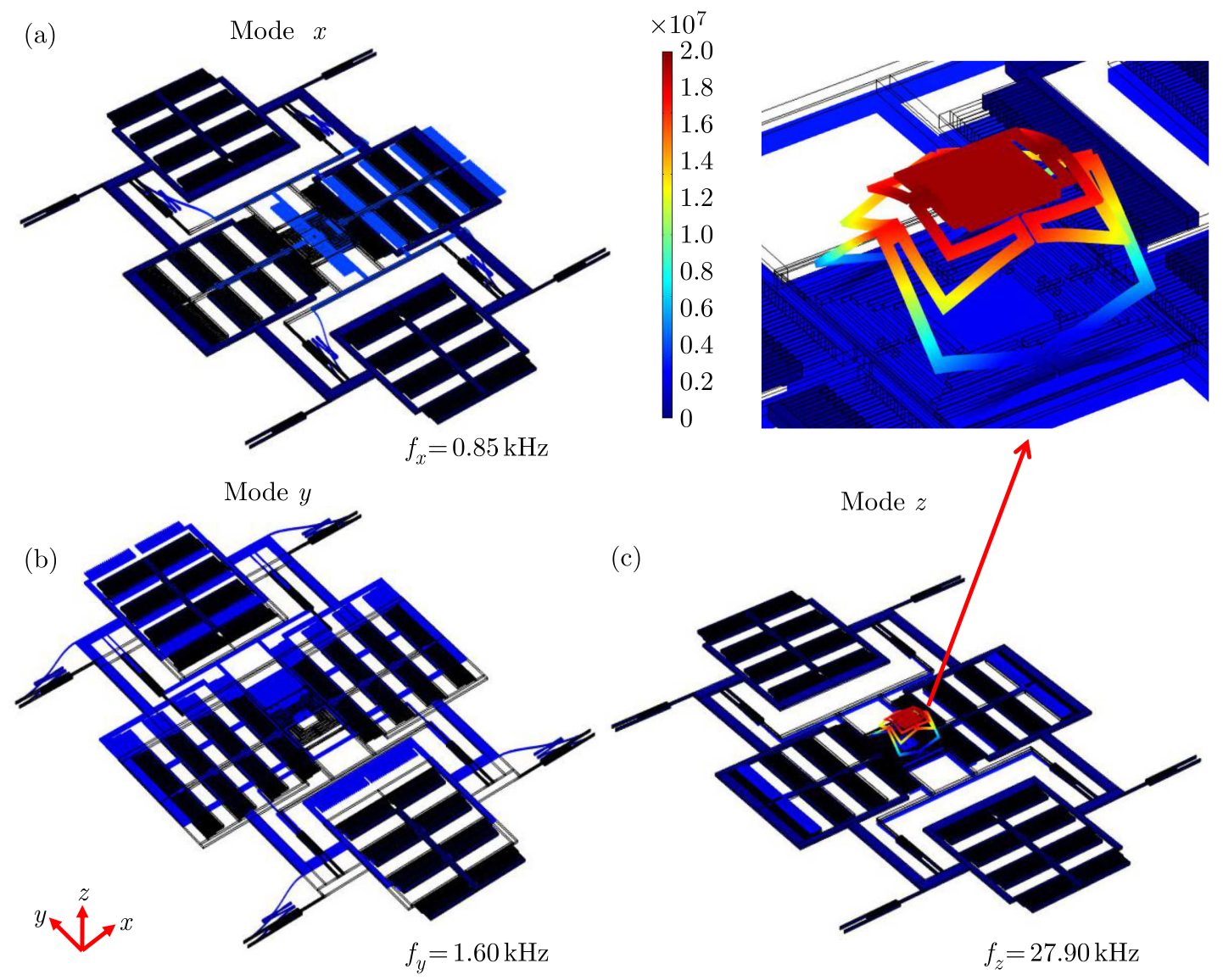

Fig. 7. The first three mode shapes and natural frequencies of the $x y z$ micro-actuator are shown in (a), (b), and (c), respectively. The dimensional parameters are shown in Table 2, in which $L=600 \mu \mathrm{m}$, $t_{x y}=30 \mu \mathrm{m}, t_{z}=10 \mu \mathrm{m}, w_{f b}=5 \mu \mathrm{m}$, and $w_{c s}=20 \mu \mathrm{m}$. The inset on the right corner shows a magnified image of the $z$ mode of the $z$-axis actuator integrated in the $x y z$ micro-actuator

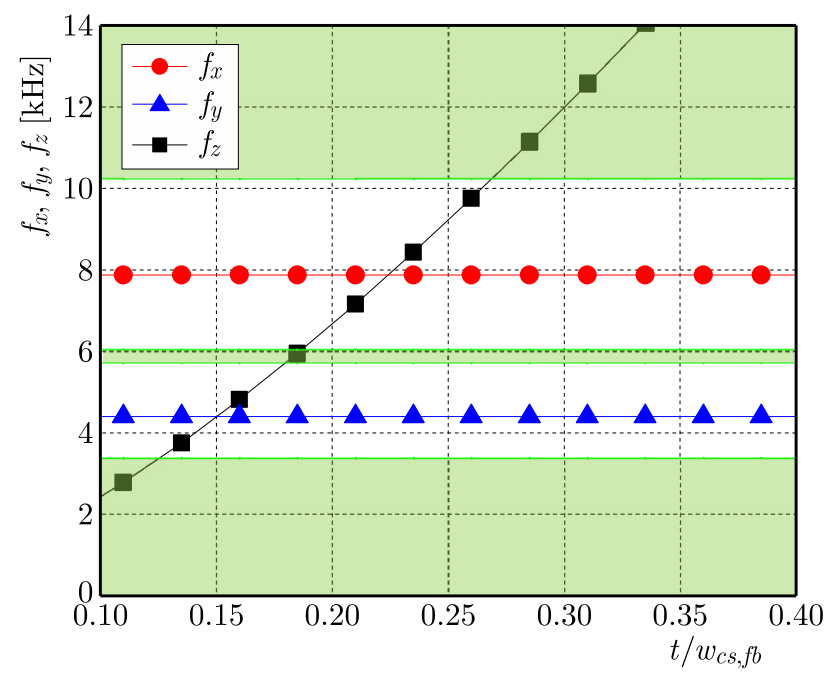

Fig. 8. The frequency of the out-of-plane mode versus the aspect ratio of $t / w_{c s, f b}$, here, $t=t_{z}=t_{x y}$

mode cross-talk $(\delta f>30 \%$ ) (Trinh et al., 2013; Weinberg and Kourepenis, 2006; Acar and Shkel, 2009 ), the operation frequency $f_{z}$ can be designed within $\left[f_{x}, f_{y}\right]$ or outside this range, as shown in green strips in Fig. 8, by modifying the parameters of the extended crab-leg beam spring as in the design of the $z$-axis actuator. The mode split between the $x$ and $y$ axes is defined 
by appropriately choosing the parameters of the folded beam spring. For example, to satisfy $\delta f>30 \%$, the ratio $t_{z} / w_{c s}$ must be $>0.27,<0.13$ or within $[0.8,0.9]$. In this study, the folded beam springs along the $x$ and $y$ axes are identical. The frequency discrepancy between $f_{x}$ and $f_{y}$ are generated from the difference in the effective mass between the $x$ and $y$ in-plane modes. To modify discrepancy in this frequency, we can choose different design parameters of the folded beam springs along the $x$ and $y$ axes.

To choose the desired operation frequency of the $x y$ actuator, we have investigated the dependence of the frequencies $f_{x}$ and $f_{y}$ and the stiffnesses $k_{x}$ and $k_{y}$ of the $x y z$ actuator on the width $w_{f b}$ of the spring beams, see Fig. 9a and $9 \mathrm{~b}$ for three thicknesses of the spring beams, $t_{x y}=10 \mu \mathrm{m}, 20 \mu \mathrm{m}$ and $30 \mu \mathrm{m}$. Figures $9 \mathrm{c}$ and $9 \mathrm{~d}$ show the frequencies $f_{x}$ and $f_{y}$ and the stiffnesses $k_{x}$ and $k_{y}$ of the $x y z$ actuator depending on the length $L$ for three widths of the spring beam, $w_{f b}=5 \mu \mathrm{m}, 7 \mu \mathrm{m}$, and $10 \mu \mathrm{m}$.

(a)

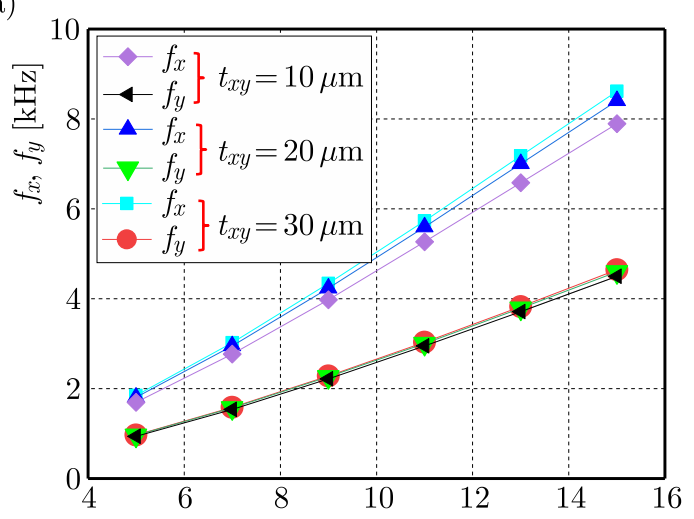

(b)

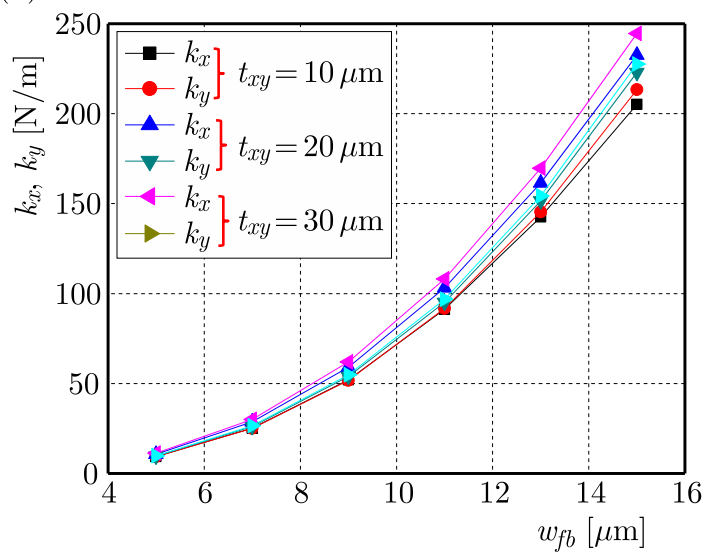

(c)

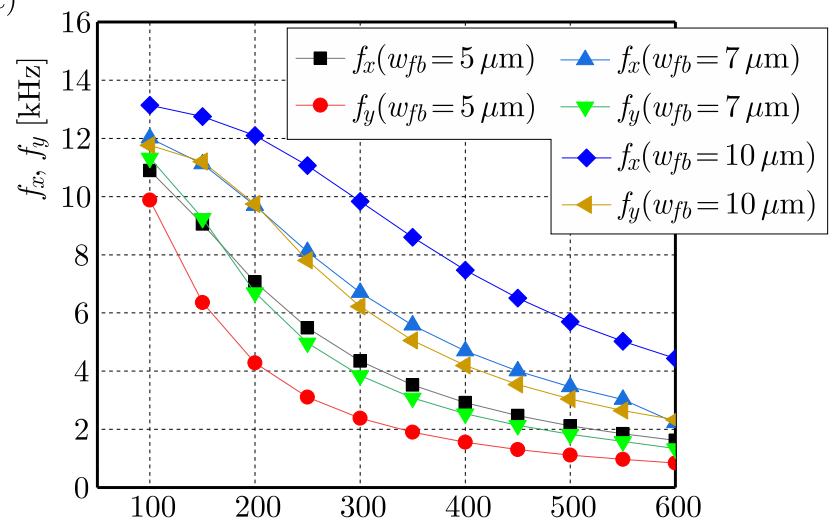

(d)

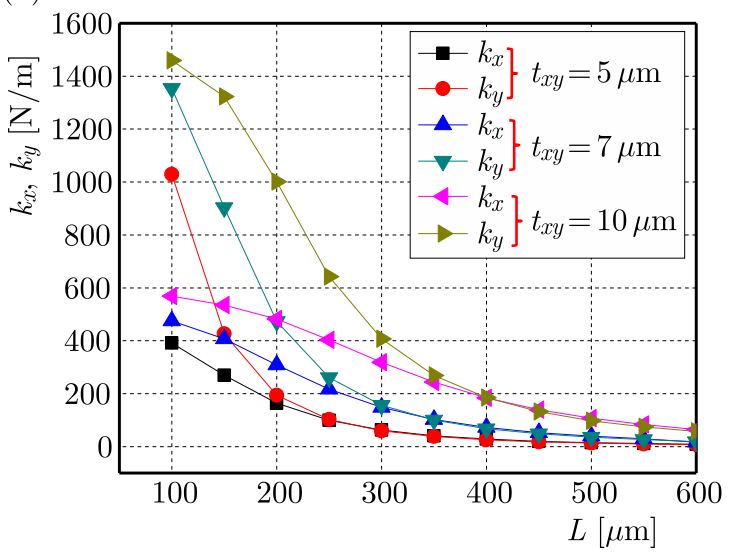

Fig. 9. Dependence of the frequencies $f_{x}$ and $f_{y}$ and the stiffnesses $k_{x}$ and $k_{y}$ of the $x y z$ actuator on the width $w_{f b}$ and length $L$ of the folded spring beam, respectively, for: (a), (b) $t_{x y}=10 \mu \mathrm{m}, 20 \mu \mathrm{m}$ and $30 \mu \mathrm{m}$ and (c), (d) $w_{f b}=5 \mu \mathrm{m}, 7 \mu \mathrm{m}$ and $10 \mu \mathrm{m}$

Thus, we can choose a wide range of the operation frequency for both $f_{x}$ and $f_{y}$. In the investigated $L$ and $w$ range, $f_{x}$ and $f_{y}$ can be varied from $1.625 \mathrm{kHz}$ to $13.141 \mathrm{kHz}$ and from $0.847 \mathrm{kHz}$ to $11.766 \mathrm{kHz}$, respectively.

The displacements of the center plate along the $x, y$ and $z$ axes are investigated as a function of applied voltage for three different gaps between the two adjacent fingers, $g_{c}=1 \mu \mathrm{m}, 1.5 \mu \mathrm{m}$ and $2 \mu \mathrm{m}$ and three actuation gaps, $g_{z}=2 \mu \mathrm{m}, 3 \mu \mathrm{m}$ and $4 \mu \mathrm{m}$ by using Eq. (3.10) and Eq. (3.8), as shown in Fig. 10a and 10b, respectively. The number of comb finger pairs in each comb drive is 160 . For the gap between comb fingers of $1 \mu \mathrm{m}$, the displacement of the plate along the $x$ and $y$ axes is $57 \mu \mathrm{m}$ for the applied voltage of $60 \mathrm{~V}$. For the $z$-axis actuator, the fixed 
electrode area is equal to the entire back-side area of the center plate. The gap between the two actuation electrodes is $3 \mu \mathrm{m}$. The maximum displacement of the center plate in this direction is $1 \mu \mathrm{m}$ with respect to the pull-in voltage of $33.8 \mathrm{~V}$ by Eq. (3.9), which is in good agreement with that obtained from simulation for $34 \mathrm{~V}$.

(a)

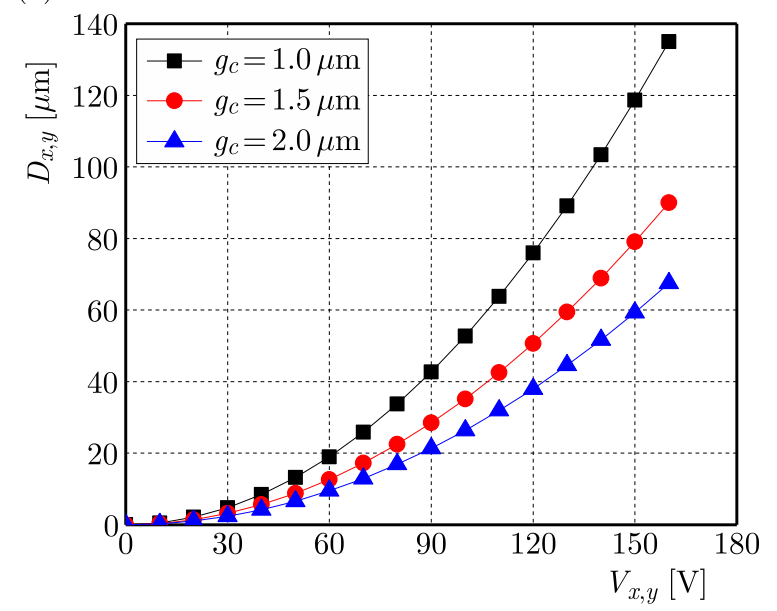

(b)

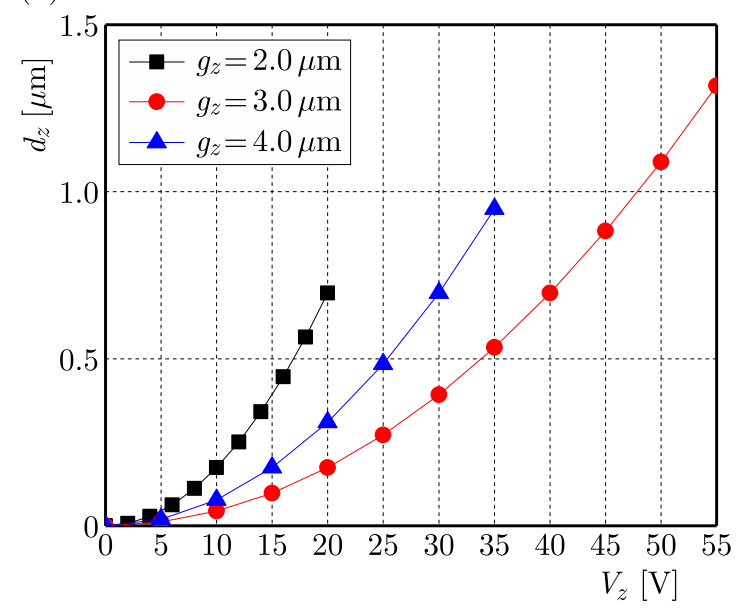

Fig. 10. The $x$ and $y$ displacements of the center plate are investigated as a function of applied voltage for three different gaps between two adjacent fingers, $g_{c}=1 \mu \mathrm{m}, 1.5 \mu \mathrm{m}$ and $2 \mu \mathrm{m}$ with $t_{x y}=30 \mu \mathrm{m}$ (a) and the $z$ displacement of the center plate is investigated as a function of applied voltage for three actuation gaps, $g_{z}=2 \mu \mathrm{m}, 3 \mu \mathrm{m}$ and $4 \mu \mathrm{m}$ with $t_{z}=10 \mu \mathrm{m}$

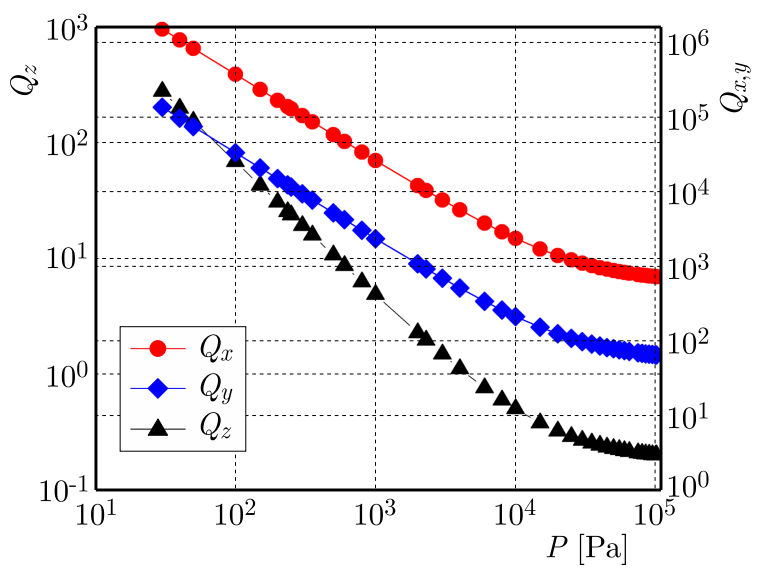

Fig. 11. Quality factors of the $x y z$ micro-actuator, $Q_{x, y, z}$, investigated as a function of ambient pressure for three vibration directions, the $x$ and $y$ axes (slide film air damping) and the $z$-axis (squeeze film air damping)

The quality factors of the $x y z$ actuator depending on ambient pressure are shown in Fig. 11. As seen in Fig. 11, the quality factor in the $z$-axis direction is lower than that compared in the $x$ and $y$ directions. This also means that damping in the $z$-axis is larger than that compared to the two remaining axes. When the ambient pressure is higher than $10^{4} \mathrm{~Pa}, Q$ is almost indepedent of $P$. For dynamic driving of the actuator, the vibration amplitue of the microactuator in each direction is calcualted by $Z=\chi Q$, in which $\chi$ is a constant depending on the dimensional parameters of the micro-actuator and the driving electrostatic force (Chu and Hane, 2011). Under the atmospheric air condition, $Q_{x, y, z}$ are calculated to be $717.4,64.7$ and 0.2 , respectively. However, at a vacuum condition of about $10^{3} \mathrm{~Pa}$, the quality factors $Q_{x, y, z}$ of the $x y z$ micro-actuator vibrating in the $x, y$ and $z$ axes are $25.7 \cdot 10^{3}, 2.3 \times 10^{3}$ and 4.87 , respectively. Therefore, if the $x y z$ micro-actuator operates under this resonant condition, the 
vibration amplitudes along the $x, y$ and $z$ axes are amplified by 35.82, 35.5 and 24.4 times, respectively.

\section{Conclusion}

We have presented design and simulation of an $x y z$ micro-actuator whose displacements can be independently controlled in three orthogonal directions. The independently controlled displacement is achieved by designing a center plate suspended by a spring system that is only compliant to the $z$ displacement. This whole plate system is suspended on a $x y$ actuator. The independent control in the three orthogonal directions is carried out by routing conductive lines along the springs and using a silicon-on-insulator wafer feed-through technique and electric isolation trenches. The optimal design of the micro-actuator is carried out by an established analysis model based on solid and fluidic mechanical theory as well as electrostatic theory. The analytical results are in good agreement with simulation results using the finite element method. The investigated results show that we can control independently the $x y z$ micro-actuator in the three orthogonal directions with a suppressed mode cross-talk. The displacements in the $x$ and $y$ directions can reach $57 \mu \mathrm{m}$ at a moderate applied voltage of $60 \mathrm{~V}$. The displacement in the $z$ direction can achieve $1 \mu \mathrm{m}$ at an applied voltage of $33.8 \mathrm{~V}$. The operation voltages of the device can be further reduced by operating it in vacuum.

\section{Acknowledgements}

This research was funded by the Hanoi University of Science and Technology (HUST) under project number T2018-PC-075.

\section{References}

1. Acar C., ShKel A., 2009, MEMS Vibratory Gyroscopes Structural Approaches to Improve Robustness, Springer Science \& Business Media LLC, USA

2. Ando Y., 2004, Development of three-dimensional electrostatic stages for scanning probe microscope, Sensors and Actuators A: Physical, 114, 2-3, 285-291

3. BAO M., 2005, Analysis and Design Principles of MEMS Devices, 1st ed., Amsterdam: Elsevier B.V.

4. Bao M., Yang H., 2007, Squeeze film air damping in MEMS, Sensors and Actuators A: Physical, 136, 3-27

5. Beer F.P., Johnston E.R., De Wolf J.T., 2003, Mechanics of Materials, Tsinghua University Press, China

6. Chu H.M., 2016, Air damping models for micro- and nano-mechanical beam resonators in molecular-flow regime, Vacuum, 126, 45-50

7. Chu H.M., Hane K., 2011, Design, fabrication and vacuum operation characteristics of two-dimensional comb-drive micro-scanner, Sensors and Actuators A: Physical, 165, 422-430

8. Chu H.M., Mizuno J., Hane K., Takagi T., 2011, Compact slanted comb two-axis micro-mirror scanner fabricated by silicon-on-insulator micromachining, Journal of Vacuum Science and Technology, B 29, 042001

9. Correa J., Koo B., Ferreira P., 2016, Parallel-kinematics XYZ MEMS. Part 1: Kinematics and design for fabrication, Precision Engineering, 46, 135-146

10. Dong J., Ferreira P.M., 2009, Electrostatically actuated cantilever with SOI-MEMS parallel kinematic XY stage, Journal of Microelectromechanical Systems, 18, 641-651 
11. Gere J.M., Timoshenko S.P., 1997, Mechanics of Materials, PWS Publishing Company

12. Hu H., Kim H., Somnath S., 2017, Tip-based nanofabrication for scalable manufacturing, $M i$ cromachines, $\mathbf{8}, 3,90$

13. Huo F., Zheng G., Liao X., Giam L.R., Chai J., Chen X., Shim W., Mirkin C.A., 2010, Beam pen lithography, Nature Nanotechnology, 5, 637-640

14. Kim Y.S., Dagalakis N.G., Gupta S.K., 2014, Design of MEMS based three-axis motion stage by incorporating a nested structure, Journal of Micromechanics and Microengineering, 24, 7, 075009

15. Legtenberg R., Groeneveld A.W., Elwenspoek M., 1996, Comb-drive actuators for large displacements, Journal of Micromechanics and Microengineering, 6, 3, 320-329

16. LiU X., KIM K., SUN Y., 2007, A MEMS stage for 3-axis nanopositioning, Journal of Micromechanics and Microengineering, 17, 9, 1796-1802

17. LiU Y., 2011, Stiffness calculation of the microstructure with crab-leg flexural suspension, Advanced Materials Research, 317-319, 1123-1126

18. Sasaki M., Bono F., Hane K., 2008, Large-displacement micro-XY-stage with paired moving plates, Japanese Journal of Applied Physics, 47, 4S, 3226

19. Takahashi K., Mita M., Fujita H., Toshiyoshi H., 2009, Switched-layer design for SOI bulk micromachined XYZ stage using stiction bar for interlayer electrical connection, Journal of Microelectromechanical Systems, 18, 4, 818-827

20. Trinh T.Q., Nguren L.Q., Dao D.V., Chu H.M., Vu H.N., 2013, Design and analysis of a $z$-axis tuning fork gyroscope with guided-mechanical coupling, Microsystem Technologies, 20, 2, 281-289

21. Veijola T., Kuisma H., Lahdenperä J., Ryhänen T., 1998, Equivalent-circuit model of the squeezed gas film in a silicon accelerometer, Sensors and Actuators A: Physical, 48, 239-248

22. Wang X., Bullen D.A., Zou J., Liu C., Mirkin C.A., 2004, Thermally actuated probe array for parallel dip-pen nanolithography, Journal of Vacuum Science and Technology, B 22, 6, 2563-2567

23. Weinberg M,S., Kourepenis A., 2006, Error sources in in-plane silicon tuning-fork MEMS gyroscopes, Journal of Microelectromechanical Systems, 15, 3, 479-491

24. Xu H., Ono T., Zhang D.Y., Esashi M., 2006, Fabrication and characterizations of a monolithic PZT microstage, Microsystem Technologies, 12, 9, 883-890

25. Zhang W.M., Yan H., Peng Z.K., Meng G., 2014, Electrostatic pull-in instability in MEMS/NEMS: A review, Sensors and Actuators A: Physical, 214, 187-218 